\title{
Effect of IL-1 Polymorphisms, CYP2C19 Genotype and Antibiotic Resistance on Helicobacter pylori Eradication Comparing Between 10-day Sequential Therapy and 14-day Standard Triple Therapy with Four-Times-Daily-Dosing of Amoxicillin in Thailand: a Prospective Randomized Study
}

\author{
Kittichet Phiphatpatthamaamphan ${ }^{1}$, Ratha-korn Vilaichone ${ }^{1,2 *}$, Sith \\ Siramolpiwat ${ }^{1}$, Anupong Tangaroonsanti ${ }^{1}$, Soonthorn Chonprasertsuk ${ }^{1}$, \\ Patommatat Bhanthumkomol $^{1}$, Bubpha Pornthisarn ${ }^{1}$, Varocha Mahachai ${ }^{2,3}$
}

\begin{abstract}
Background: Studies of effects of IL-1 polymorphisms, CYP2C19 genotype together with antibiotic resistance for $H$. pylori eradication are rare worldwide. The present study was designed to evaluate efficacy of 10-day sequential therapy (SQT) and 14-day standard triple therapy (STT) with four- times-daily dosing of amoxicillin for $\mathrm{H}$. pylori eradication related to these important host and bacterial factors in Thailand. Materials and Methods: This prospective randomized study was performed during March 2015 to January 2016. H. pylori infected gastritis patients were randomized to receive 10-day sequential therapy and 14-day standard triple therapy. CYP2C19 genotyping, IL1 polymorphism (IL-1B and IL-1RN genotypes) and antibiotic susceptibility tests were performed in all patients. 13C-UBT was conducted to confirm $H$. pylori eradication at least 4 weeks after treatment. Results: $A$ total of 100 patients ( 33 males and 67 females, mean age=51.1 years) were enrolled. Eradication rate by PP analysis was $97.9 \%(47 / 48)$ with the 10-day SQT regimen and $87.8 \%(43 / 49)$ with 14-day STT regimen $(97.9 \%$ vs $87.8 \%$; p-value $=\mathbf{0 . 0 5 3}$ ). Antibiotic susceptibility testing demonstrated $45 \%$ resistance to metronidazole, $14.8 \%$ to clarithromycin, and $24.1 \%$ to levofloxacin. CYP2C19 genotyping revealed $44.9 \%$ RM, $49 \%$ IM and $6.1 \%$ PM.IL-1B and IL-1RN genotypes were demonstrated as $21.4 \%$ for $\mathrm{CC}, \mathbf{4 8 . 1 \%}$ for TC, 36.8\% for TT, $72.7 \%$ for $1 / 1$, and $21.2 \%$ for $1 / 2$ genotypes, respectively. The 10-day SQT regimen provided $100 \%$ eradication in patients with clarithromycin or dual clarithromycin and levofloxacin $H$. pylori resistant strains. Moreover, the 10-day SQT regimen resulted in a $100 \%$ eradication rate in all patients with CYP2C19 genotype $R M$ and almost type of IL-1B (TC and TT) and IL1-RN genotypes ( 1/2 and other). Conclusions: Treatment with 10-day sequential therapy is highly effective for $\mathrm{H}$. pylori eradication regardless of the effects of clarithromycin resistance, dual clarithromycin and levofloxacin resistance, CYP2C19 genotype, IL-1B and IL1-RN genetic polymorphisms and can be used as effective first line therapy in Thailand.
\end{abstract}

Keywords: IL-1 polymorphisms - dosing scheme of amoxicillin triple therapy -10-day sequential therapy

Asian Pac J Cancer Prev, 17 (4), 1903-1907

\section{Introduction}

Helicobacter pylori (H. pylori) infection is the main cause of peptic ulcer disease (PUD), mucosa associated lymphoid tissue lymphoma, and gastric cancer (Vilaichone and Mahachai, 2001; Vilaichone et al., 2006). Furthermore, $H$. pylori eradication potentially reduces the incidence of gastric cancer, especially in high prevalence area of this particular cancer (Ford et al., 2014; Demirel et al., 2013). However, the success of eradication regimens depends on many factors including compliance, antibiotic resistance, and proper dosing of proton pump inhibitor (PPI) that provides adequate acid suppression (Mahachai et al., 2011)

The global eradication rate of standard triple regimen for $H$. pylori infection has declined in recent years and no longer recommended as an empiric choice in most countries (Chey and Wong, 2007; Mahachai et al., 2011). However, an extended duration of PPI and clarithromycincontaining triple therapy from 7 to 10-14 days improved the eradication rate by about $5-10 \%$ (Vilaichone et al,

${ }^{1}$ Gastroenterology Unit, Thammasat University Hospital, Pathumthani, ${ }^{2}$ National Gastric Cancer and Gastrointestinal Diseases Research Center, ${ }^{3}$ Gastrointestinal and Liver Center, Bangkok Medical Center, Bangkok, Thailand *For correspondence: Vilaichone@ hotmail.co.th 
Kittichet Phiphaaamphan et al

2006, Malfertheiner et al., 2012). Interestingly, recent study has demonstrated the positive effect of the dosing scheme of amoxicillin significantly influenced eradication rates of triple therapies. Eradication rates in regimens with tid and qid dosing of amoxicillin were higher than those of regimens with the bid dosing of amoxicillin (Furuta et al., 2014). One of the popular present first-line treatment options is sequential regimen, which has shown higher eradication rates than conventional triple therapy in several recent meta-analyses (Zullo et al., 2007; Jafri et al., 2008; Gatta et al., 2009). IL-1 polymorphisms and CYP2C19 genotype together with antibiotic resistance of $H$. pylori markedly affect the eradication rate but rarely reports worldwide.

We conduct this study for evaluated efficacy of 10day sequential therapy (SQT) and 14-day standard triple therapy (STT) with four times-daily dosing of amoxicillin for H.pylori eradication in Thai patients with functional dyspepsia as a first line H. pylori eradication in Thailand. We also evaluated antibiotic resistance pattern as well as the effect of IL-1 polymorphisms and CYP2C19 genotype with these two regimens.

\section{Materials and Methods}

\section{Patients}

Eligible patients age between 18-70 years who underwent gastroscopic examination at Thammasat University Hospital, Pathumthani, Thailand for dyspeptic symptoms during March 2015 and January 2016 were enrolled. After the endoscopy, those with diagnosis of non-ulcer dyspepsia, which was established during gastroscopy with normal finding or mild gastritis, were considered entering in this study. Exclusion criteria included patients with (1) a history of previous $H$. pylori eradication, (2) previous gastric surgery (3) currently on anticoagulants drugs, (4) administration of antibiotics, bismuth and PPI drugs in the preceding 4 weeks, (5) pregnancy, breast-feeding woman, (6) allergy to any one of the given medication in the regimens, and (7) other serious comorbidities (organ failure, malignancies). All patients provided written informed consent.

\section{The diagnosis of $H$. pylori infection}

During the endoscopy, 4 biopsy samples from gastric antrum were obtained for rapid urease test, H. pylori culture and Epsilometer test (E-test) or GenoType ${ }^{\circledR}$ HelicoDR, histological examination, IL-1 polymorphisms and CYP2C19 genotype. The results of CYP2C19 genotype testing were expressed as: rapid metabolizer (RM), intermediate metabolizer (IM) or poor metabolizer (PM). The results of IL1 polymorphisms (IL$1 \mathrm{~B}$ and IL-1RN genotypes) testing was expressed as: CC, TC, TT, 1/1, 1/2, and other genotypes, respectively. The presence of $H$. pylori was defined as: (1) positive $H$. pylori culture, or (2) positive tests (rapid urease test or histology).

\section{Therapeutic regimens}

Randomization was made by reference to a computergenerated list. The two different groups were given (1) 10-day SQT (rabeprazole $20 \mathrm{mg}$, amoxicillin 1,000 mg twice daily for 5 days, followed by rabeprazole $20 \mathrm{mg}$ twice daily, $1 \mathrm{~g}$ long acting clarithromycin MR once daily, and metronidazole $400 \mathrm{mg}$ three times-daily for 5 days) (2) 14-day STT with four times-daily dosing of amoxicillin (rabeprazole $20 \mathrm{mg}$ twice daily, amoxicillin $500 \mathrm{mg}$ four times-daily, and $1 \mathrm{~g}$ long acting clarithromycin MR once daily for 14 days)

\section{Post-therapy follow-up}

After completion of therapy for at least 4 weeks, 13C-urea breath test (UBT) was performed for all individuals to determine $H$. pylori eradication. Successful eradication was defined as negative result from 13C-UBT. Pill count was observed, and drug consumption over $90 \%$ was defined as good adherence. Side effects were assessed by personal interview using questionnaires for each patient. The potential adverse reactions listed in the questionnaires were bitter taste, nausea and vomiting, palpitation, diarrhea, and skin rashes. New symptoms and exacerbation of pre-existing symptoms during the period of treatment were determined to be therapy-related adverse reactions. Serious adverse reactions were defined as symptoms that disturbed on patients' daily life.

\section{Statistical analysis}

We expected the eradication rate of 14-day STT with four times-daily dosing of amoxicillin as an empiric therapy to be $\geq 90 \%$. Treatment success was defined as a cure rate of $\geq 95 \%$ (i.e. grade A) as described in prior study (Graham et al., 2009), and failure as a cure rate of $<90 \%$ per protocol analyses. The demographic findings and frequencies of adverse events were compared using chisquared, Fisher's exact and student's t-test. P-value $<0.05$ was considered to be statistically significant. The study was conducted according to good clinical practice guideline, as well as the Declaration of Helsinki, and was also approved by our local ethics committee.

\section{Results}

Total of 100 patients were included in this study, 33 men and 67 women with a mean age of 51.1 years. All 100 patients were randomized in to 2 groups as previously described. The patients with 10-day SQT regimens were younger age than those of 14-day STT group. Others baseline demographic data are shown in Table 1. One

Table 1. Baseline Demographic Data of All Patients

\begin{tabular}{lccc}
\hline \multicolumn{1}{c}{ Characteristics } & $\begin{array}{c}14 \text {-day STT } \\
(50)\end{array}$ & $\begin{array}{c}10 \text {-day SQT } \\
(50)\end{array}$ & P-value \\
\hline Age (yr) [range] & $53.6[29-70]$ & $48.5[22-69]$ & \\
Sex(N) male & $17(34 \%)$ & $16(32 \%)$ & 0.83 \\
female & $33(66 \%)$ & $34(68 \%)$ & \\
Comorbidity dis. & & & \\
T2DM & $5(10 \%)$ & $3(6 \%)$ & 0.46 \\
HTN & $14(28 \%)$ & $8(16 \%)$ & 0.15 \\
DLP & $9(18 \%)$ & $8(16 \%)$ & 0.79 \\
H/O smoking & $1(2 \%)$ & $2(4 \%)$ & 0.56 \\
H/O alcohol & $2(4 \%)$ & $3(6 \%)$ & 0.64 \\
consumption & & & \\
\hline
\end{tabular}


IL-1 Polymorphisms, CYP2C19 Genotype and Antibiotic Resistance for Helicobacter pylori Eradication in Thailand

patient in 14-day STT group was loss to follow up after complete treatment due to poor adherence. Two patients in 10-day SQT regimen were loss to follow up due to poor adherence and concerning about side effects of treatment regimen.

\section{Eradication rate of treatment regimens}

The eradication rates were calculated by both intention-to-treat (ITT) and per-protocol (PP) analyses as shown in Figure 1. The eradication rates by ITT analysis was $86 \%$ (43/50) with 14-day STT regimen and 94\% (47/50) with 10-day SQT regimen. PP eradication results were $87.8 \%$ (43/49) and $97.9 \%$ (47/48) with 14-day STT and 10-day SQT regimen, respectively. The cure rate was higher in 10-day SQT regimen than those in 14-day STT regimen from both ITT and PP. Only PP eradication of 10-day SQT group could achieve cure rate of grade A $(\geq 95 \%)$ as detail in Figure 1 .

Antibiotic susceptibility and H. pylori eradication rate

Antibiotic susceptibility tests were performed in 54 strains (40 from E-test and 14 from GenoType $® H e l i c o D R$ ), which demonstrated $45 \%$ of metronidazole resistant, $24.1 \%$ of levofloxacin resistant and $14.8 \%$ of clarithromycin resistant strains as detail in Figure 2. The 10-day SQT regimen provided $100 \%$ eradication in patients with clarithromycin or dual clarithromycin \& levofloxacin $H$. pylori resistant strains as detail in Table 2.

\section{Host factors and H. pylori eradication rate}

CYP2C19 genotype tests were performed in 98 cases (48 from 14-day STT regimen and 50 from10-day SQT regimen). The CYP2C19 genotype tests revealed $44.9 \%$ RM, 49\% IM and 6.1\% PM. The prevalence of CYP2C19

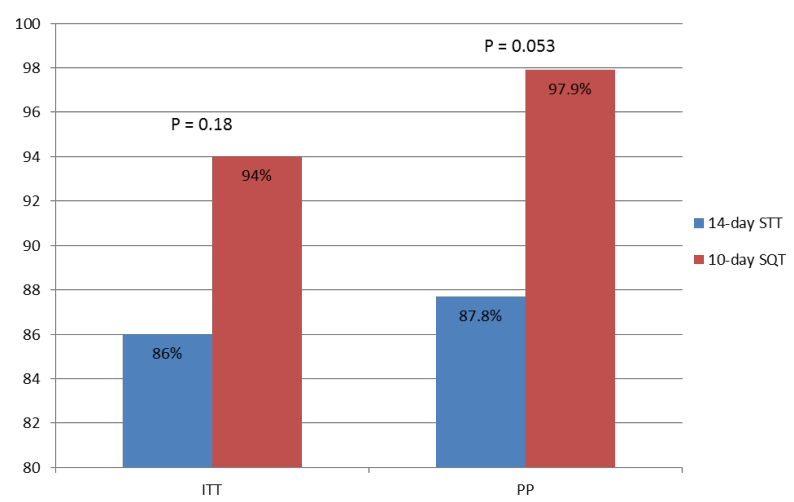

Figure 1. Eradication Rates with Both Regimens

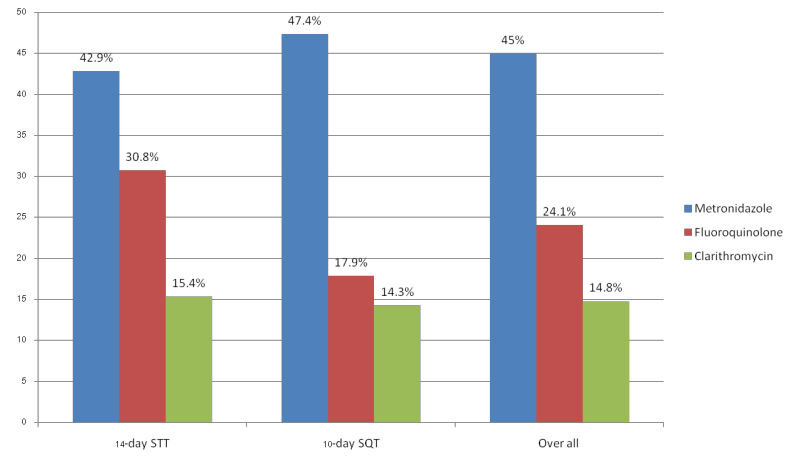

Figure 2. Prevalence of Antibiotic Resistance genotype was similar in both groups as shown in Table 3.

IL-1B genotype were performed in 98 cases (48 from 14-day STT regimen and 50 from10-day SQT regimen) and revealed $21.4 \% \mathrm{CC}, 41.8 \% \mathrm{TC}$ and $36.8 \% \mathrm{TT}$. IL$1 \mathrm{RN}$ genotype was also performed in 99 cases (49 from 14-day STT regimen and 50 from10-day SQT regimens) and demonstrated $72.7 \%$ for genotype $1 / 1,21.2 \%$ for genotype $1 / 2$ and $6.1 \%$ for other genotypes. The result of IL-1 polymorphism and eradication rate were similar in all groups of patients. The 10-day SQT regimen has 100\% eradication rate in all patients with CYP2C19 genotype RM, IM and almost type of IL-1B and IL1-RN genotypes as detailed in Tables 4 and 5.

\section{Adverse events}

The common side effects include bitter taste, nausea/ vomiting, abdominal pain, diarrhea, headache, and fatigue which were found in all groups. Documented adverse reactions are shown in Table 6 . None of subject experienced any major adverse event.

Table 2. Antibiotic Susceptibility and Eradication Rates for Each Regimen

\begin{tabular}{lccc}
\hline $\begin{array}{c}\text { Antibiotic susceptibility } \\
\text { test }\end{array}$ & 14-day STT & $\begin{array}{c}10 \text {-day } \\
\text { SQT }\end{array}$ & $\begin{array}{c}\text { P- } \\
\text { value }\end{array}$ \\
\hline $\begin{array}{l}\text { Clarithromycin (C) } \\
\text { resistance }\end{array}$ & $2 / 4(50 \%)$ & $3 / 3(100 \%)$ & 0.15 \\
$\begin{array}{l}\text { Metronidazole (M) } \\
\text { resistance }\end{array}$ & $8 / 9(88.9 \%)$ & $9 / 9(100 \%)$ & 0.3 \\
Fluoroquinolone (F) & $6 / 8(75 \%)$ & $5 / 5(100 \%)$ & 0.27 \\
resistance & $1 / 2(50 \%)$ & $0 / 0(0 \%)$ & 0.12 \\
C + M resistance & $0 / 0(0 \%)$ & $2 / 2(100 \%)$ & 0.12 \\
C + F resistance & $2 / 2(100 \%)$ & $1 / 1(100 \%)$ & 0.12 \\
M + F resistance & $0 / 0(0 \%)$ & $0 / 0(0 \%)$ & \\
C + M + F resistance & & & \\
\hline
\end{tabular}

Table 3. CYP2C19 Genotypes and Eradication Rates

\begin{tabular}{cccc}
\hline $\begin{array}{c}\text { CYP2C19 genotype } \\
(\mathrm{N}=98)\end{array}$ & $\begin{array}{c}\text { 14-day } \\
\text { STT }(48)\end{array}$ & $\begin{array}{c}\text { 10-day } \\
\text { SQT }(50)\end{array}$ & P-value \\
\hline RM $(\mathrm{N}=44 ; 44.9 \%)$ & $25(92 \%)$ & $19(100 \%)$ & 0.16 \\
$\mathrm{IM}(\mathrm{N}=48 ; 49 \%)$ & $21(81 \%)$ & $27(100 \%)$ & 0.31 \\
$\mathrm{PM}(\mathrm{N}=6 ; 6.1 \%)$ & $2(100 \%)$ & $4(75 \%)$ & 0.43 \\
\hline
\end{tabular}

Table 4. IL-1B Genotypes and Eradication Rates

\begin{tabular}{cccc}
\hline IL1B (N=98) & $\begin{array}{c}\text { 14-day } \\
\text { STT (48) }\end{array}$ & $\begin{array}{c}\text { 10-day SQT } \\
(50)\end{array}$ & P-value \\
\hline CC $(\mathrm{N}=21 ; 21.4 \%)$ & $7(85.7 \%)$ & $14(93 \%)$ & 0.11 \\
TC $(\mathrm{N}=41 ; 41.8 \%)$ & $20(80 \%)$ & $21(100 \%)$ & 0.97 \\
TT $(\mathrm{N}=36 ; 36.8 \%)$ & $21(95 \%)$ & $15(100 \%)$ & 0.16 \\
\hline
\end{tabular}

Table 5. IL-1 RN Genotypes and Eradication Rates

\begin{tabular}{cccc}
\hline IL1RN (N=99) & $\begin{array}{c}\text { 14-day STT } \\
(49)\end{array}$ & $\begin{array}{c}\text { 10-day SQT } \\
(50)\end{array}$ & P-value \\
\hline $1 / 1(\mathrm{~N}=72 ; 72.7 \%)$ & $38(83.8 \%)$ & $34(96.9 \%)$ & 0.29 \\
$1 / 2(\mathrm{~N}=21 ; 21.2 \%)$ & $9(100 \%)$ & $12(100 \%)$ & 0.49 \\
Other $(\mathrm{N}=6 ; 6.1 \%)$ & $2(100 \%)$ & $4(100 \%)$ & 0.41 \\
\hline
\end{tabular}


Table 6. Adverse Events with Each Regimen

\begin{tabular}{lccc}
\hline $\begin{array}{c}\text { Adverse events } \\
(\mathrm{N}=97)\end{array}$ & $\begin{array}{c}\text { 14-day STT } \\
(49)\end{array}$ & $\begin{array}{c}\text { 10-day SQT } \\
(48)\end{array}$ & P-value \\
\hline Bitter taste & $18(36.7 \%)$ & $15(31.3 \%)$ & 0.57 \\
Nausea/vomiting & $9(18.4 \%)$ & $13(27.1 \%)$ & 0.31 \\
Abdominal pain & $8(16.3 \%)$ & $12(25 \%)$ & 0.29 \\
Diarrhea & $6(12.2 \%)$ & $8(16.7 \%)$ & 0.54 \\
Headache & $5(10.2 \%)$ & $4(8.3 \%)$ & 0.75 \\
Fatigue & $4(8.2 \%)$ & $8(16.7 \%)$ & 0.2 \\
\hline
\end{tabular}

\section{Discussion}

Currently, the eradication rate of $H$.pylori by STT was reported to be less than $70 \%$ in many countries worldwide, including Thailand (Vilaichone et al., 2006; Graham, 2009). Recent studies from Japan have demonstrated that the eradication rates in clarithromycin-based triple regimens with qid dosing of amoxicillin were higher than those of regimens with the bid dosing of amoxicillin about $14 \%$ (91.9\% and $77.8 \%$ respectively.) (Furuta et al., 2014). The data on the effectiveness of the dosing scheme of amoxicillin triple therapy with clarithromycin-containing regimen in Thailand are limited. However, we reported the effectiveness of 14-day STT with four times-daily dosing of amoxicillin were only $86 \%$ by ITT and $87.8 \%$ by PP analyses which failed to achieve grade A eradication rate $(>95 \%)$ and insufficient to be the first line therapy regimen in Thailand.

SQT has been tested mainly in Europe and found to have higher eradication rate than STT (Zullo et al., 2007). Recent study from Thailand has shown that a 10-day SQT is highly effective and well tolerated with $95 \%$ eradication rate. However, the eradication rate was drop to $57.1 \%$ with clarithromycin-resistant strains (Sirimontaporn et al., 2010). Our study has demonstrated that the efficacy of 10-day SQT were as high as $94 \%$ by ITT and $97.9 \%$ by PP analyses and could overcome the effect clarithromycinresistant and dual clarithromycin-fluoroquinolone resistant strains. Metronidazole resistance has been reported approximately $35-50 \%$ in Thailand (Vilaichone et al, 2013; Srinarong et al, 2014; Prapitpaiboon et al, 2015; Vilaichone et al, 2015 ). Interestingly, 10-day SQT has also shown to overcome this common antibiotic resistant strain of $H$. pylori infection. Larger clinical trials need to confirm these important findings.

CYP2C19 genotype has recently been found to have an impact on $H$. pylori eradication, peptic ulcer healing, and therapeutic efficacy of PPI (Furuta et al., 2001; Yamada et al., 2001). This study demonstrated that genotype IM was the most common genotype same as prior studies in Thailand (Jainan et al, 2014; Srinarong et al, 2014). IL-1 polymorphisms (IL-1B and IL-1RN) have been consistent to increase gastric mucosal inflammation related to $H$. pylori infection by influenced gastric mucosal cytokine levels (Vilaichone et al., 2005). Rarely prior studies reported effect of IL-1 polymorphisms and CYP2C19 genotype and H. pylori eradication rate worldwide. Interestingly, the 10-day SQT regimen has high eradication rate $(100 \%)$ in genotypes RM and IM of CYP2C19 and most genotype of IL-1polymorphisms.
In summary, our study demonstrated high eradication rate (grade A) of $H$. pylori infection from 10-day SQT regardless of CYP2C19 genotype, clarithromycin or dual clarithromycin and fluoroquinolone resistant strains. Only minor side effects were observed from this regimen. Our study supports the idea that 10-day SQT can be used as effective first line therapy for $H$. pylori eradication in Thailand.

\section{Acknowledgements}

This study was partially supported by Research Fund at Faculty of Medicine, Thammasat University Hospital, Thailand, and Gastroenterology Association of Thailand (GAT).

\section{References}

Chey WD, Wong BC (2007). American College of Gastroenterology guideline on the management of Helicobacter pylori infection. Am J Gastroenterol, 102, 1808-25.

Demirel BB, Akkas BE, Vural GU (2013). Clinical factors related with helicobacter pylori infection - is there an association with gastric cancer history in first- degree family members? Asian Pac J Cancer Prev, 14, 1797-1802.

Ford AC, Forman D, Hunt RH, et al (2014). Helicobacter pylori eradication therapy to prevent gastric cancer in healthy asymptomatic infected individuals: systematic review and meta-analysis of randomised controlled trials. $B M J, \mathbf{3 4 8}$, 3174.

Furuta T, Shirai N, Takashima M, et al (2001). Effect of genotypic differences in CYP2C19 on cure rates for Helicobacter pylori infection by triple therapy with a proton pump inhibitor, amoxicillin, and clarithromycin. Clin Pharmacol Ther, 69, 158-68.

Furuta T, Sugimoto M, Yamade M, et al (2014). Effect of dosing schemes of amoxicillin on eradication rates of Helicobacter pylori with amoxicillin-based triple therapy. $J$ Clin Pharmacol, 54, 258-66.

Gatta L, Vakil N, Leandro G, et al (2009). Sequential therapy or triple therapy for Helicobacter pylori infection: systematic review and meta-analysis of randomized controlled trials in adults and children. Am J Gastroenterol, 104, 3069-79.

Graham DY (2009). Efficient identification and evaluation of effective Helicobacter pylori therapies. Clin Gastroenterol Hepatol, 7, 145-8.

Jafri NS, Hornung CA, Howden CW, (2008). Meta-analysis: sequential therapy appears superior to standard therapy for Helicobacter pylori infection in patients naive to treatment. Ann Intern Med, 148, 923-31.

Jainan W, Vilaichone RK (2014). Effects of the CYP2C19 genetic polymorphism on gastritis, peptic ulcer disease, peptic ulcer bleeding and gastric cancer. Asian Pac J Cancer Prev, 15, 10957-60

Mahachai V, Sirimontaporn N, Tumwasorn S, et al (2011). Sequential therapy in clarithromycin-sensitive and -resistant Helicobacter pylori based on polymerase chain reaction molecular test. J Gastroenterol Hepatol, 26, 825-8.

Malfertheiner P, Megraud F, O'Morain CA, et al (2012). Management of Helicobacter pylori infection--the maastricht iv/ florence consensus report. Gut, 61, 646-64.

Prapitpaiboon H, Mahachai V, Vilaichone RK (2015). High efficacy of levofloxacin-dexlansoprazole-based quadruple therapy as a first line treatment for Helicobacter pylori 
IL-1 Polymorphisms, CYP2C19 Genotype and Antibiotic Resistance for Helicobacter pylori Eradication in Thailand eradication in Thailand. Asian Pac J Cancer Prev, 16, 4353-6.

Sirimontaporn N, Thong-Ngam D, Tumwasorn S, et al (2010). Ten-day sequential therapy of Helicobacter pylori infection in Thailand. Am J Gastroenterol, 105, 1071-5.

Srinarong C, Siramolpiwat S, Wongcha-um A, et al (2014). Improved eradication rate of standard triple therapy by adding bismuth and probiotic supplement for Helicobacter pylori treatment in Thailand. Asian Pac J Cancer Prev, 15, 9909-13.

Vilaichone RK, Gumnarai P, Ratanachu-Ek T, et al (2013). Nationwide survey of Helicobacter pylori antibiotic resistance in Thailand. Diagn Microbiol Infect Dis, 77, 346-9.

Vilaichone RK, Mahachai V (2001). Current management of Helicobacter pylori infection. J Med Assoc Thai, 84, 32-38.

Vilaichone RK, Mahachai V, Tumwasorn S, et al (2005). Gastric mucosal cytokine levels in relation to host interleukin-1 polymorphisms and Helicobacter pylori cagA genotype. Scand J Gastroenterol, 40, 530-39

Vilaichone RK, Mahachai V, Graham DY (2006). Helicobacter pylori diagnosis and management. Gastroenterol Clin North Am, 35, 229-47.

Vilaichone RK, Prapitpaiboon H, Gamnarai P, et al (2015). Seven-day bismuth-based quadruple therapy as an initial treatment for Helicobacter pylori infection in a high metronidazole resistant area. Asian Pac J Cancer Prev, 16, 6089-6092.

Yamada S, Onda M, Kato S, et al (2001). Genetic differences in CYP2C19 single nucleotide polymorphisms among four Asian populations. J Gastroenterol, 36, 669-72.

Zullo A, De Francesco V, Hassan C, et al (2007). The sequential therapy regimen for Helicobacter pylori eradication: a pooled-data analysis. Gut, 56, 1353-7. 